\section{Upper Gastrointestinal Bleeding and Intestinal Perforation Due to Multiple Duodenojejunal Metastases from a Silent Bronchogenic Adenosquamous Carcinoma}

We report here the case of a 59-year-old man, a heavy smoker, who presented with epigastric pain, postprandial nausea, weight loss, and intermittent melena. Bilateral pulmonary fibrosis had been diagnosed when he was aged 40 ; otherwise he had a negative medical history.

"Volcano-like" lesions with tip hemorrhage and polypoid masses of varying size $(2-20 \mathrm{~mm}$ in diameter) involving the second portion of the duodenum were found at panendoscopy (Figures 1,2). The biopsy samples were interpreted as metastatic adenocarcinoma in the duodenum. A small adenosquamous carcinoma in one of the right segmental bronchi was identified as the primary neoplasm during a systematic survey including thoracic computed tomography (CT) and bronchoscopy. Biopsies obtained from the bronchogenic cancer were histologically similar to the duodenal ones. An abdominal CT showed further metastases in both adrenal glands. The patient's anemia became rapidly worse in spite of massive blood transfusion and hemostatic treatment. Three weeks after admission, he underwent surgery due to jejunal perforation caused by multiple metastatic tumor masses involving the second portion of the duodenum and the proximal part of the jejunum. The patient died the day after surgery.

Metastatic tumors of the upper gastrointestinal tract are rare. Most of them are postmortem findings, and they are rarely of clinical significance (1). Metastases involving the upper gastrointestinal tract that can be evaluated endoscopically occur mainly in patients with a known primary malignancy. In this case, the duodenal metastases were the first clinical symptoms of the disease. As described in previous reports, metastases identified by endoscopy and histology did not provide any specific information that would allow the primary cancer to be predicted $(2,3)$. Duodenojejunal metastases from a primary bronchogenic tumor are extremely rare, but should be included in the differential diagnosis of gastrointestinal bleeding in patients with a known bronchogenic tumor. In view of the current incidence of lung cancer, further patients can be expected

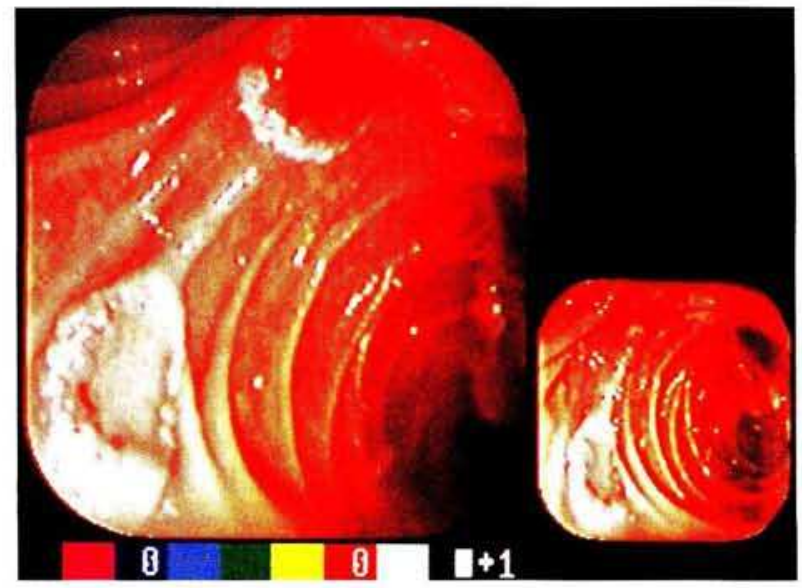

Figure 1: "Volcanolike" lesions in the second part of the duodenum.

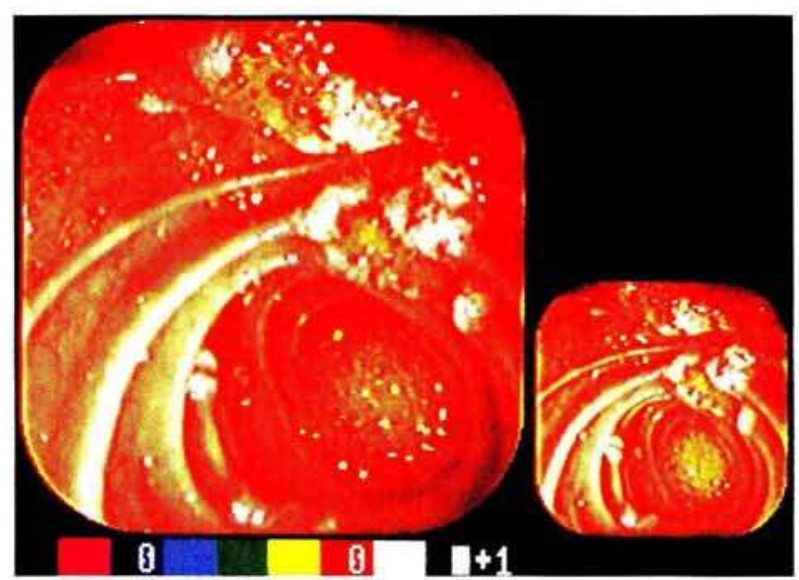

Figure 2: Polypoid masses of various sizes in the second portion of the duodenum.

with abdominal complaints secondary to metastatic disease of the upper gastrointestinal tract.

M. Fischer', J. Papp', J. Kulka',

M. Zsiray ${ }^{3}$, P. Kempler ', F Szalay

' First Dept. of Medicine

${ }^{2}$ Second Dept. of Pathology

${ }^{3}$ Dept. of Pulmonology,

Semmelweis University Medical School, Budapest, Hungary

\section{References}

1. Telerman A, Gerard B, Van den Heule $B$, et al. Gastrointestinal metastasis from extra-abdominal tumors. Endoscopy $1985 ; 17: 99-101$.
2. Hsu CC, Chen JJ, Changchien CS Endoscopic features of metastatic tumors in the upper gastrointestinal tract. Endoscopy 1996; 28: 249-53.

3. Morris DM, Deitch EA. Clinically significant intestinal metastasis from a primary bronchogenic carcinoma. J Surg Oncol 1983; 23: $93-4$

Corresponding Author M. Fischer, M.D

First Dept. of Medicine Semmelweis University Medical School

Korányi S. u. 2/a

1083 Budapest, Hungary

Fax: + 36-1-210-0279 